\title{
Telemedicina e sua relação com comunicação, tecnologia e convergência
}

\section{Telemedicine and its relationship with communication, technology and convergence}

\author{
Rosemary de Carvalho Rocha Koga ${ }^{1}$, José Raimundo da Silva Koga ${ }^{2}$ \\ ${ }^{1}$ Biomédica, Doutoranda em Inovação Farmacêutica pela Universidade Federal do Amapá - UNIFAP. Macapá-AP Brasil. E-mail: \\ rosemarykoga@gmail.com *Autor para correspondência \\ ${ }^{2}$ Acadêmico de Jornalismo. Universidade Federal do Amapá - UNIFAP. Macapá-AP Brasil. E-mail: jrskoga@gmail.com
}

\author{
Palavras-chave \\ Telemedicina \\ Convergência \\ Tecnologia \\ Comunicação e Saúde
}

\begin{abstract}
Este artigo discorre sobre o papel do profissional jornalista em promover a informação em saúde, principalmente em serviço de telemedicina. Questiona-se, via cultura da convergência, como a comunicação pode contribuir no serviço de telemedicina? Como a inteligência coletiva por meio de experiências reais de vida pode colaborar na convergência da medicina tradicional para a telemedicina? Em paralelo a visão defendida por Henry Jenkins (2009) quando define meios de comunicação, observa-se uma mudança de protocolos das pessoas que buscam esclarecimentos sobre saúde. Supõe-se que essas mudanças de protocolo contribuem para desdobramentos de tratamentos e da saúde geral dos pacientes. Este trabalho resume por meio de pesquisa bibliográfica conceitos em telemedicina abordados por diversos autores. Espera-se, pela cultura da convergência, a autonomia do paciente, como por exemplo, em escolher qual forma deseja ser consultado, qual método que lhe oferece menor risco e maior benefício de saúde.
\end{abstract}

\begin{abstract}
Keywords
Telemedicine

Convergence

Technology

Communication and Health

This article discusses the role of the professional journalist in promoting health information, especially in telemedicine services. It is questioned, via the convergence culture, how can communication contribute to the telemedicine service? How can collective intelligence through real life experiences collaborate in the convergence of the traditional medicine to telemedicine? In parallel with the view defended by Henry Jenkins (2009) when defining means of communication, there is a change in the protocols of people who seek clarification about health. It is assumed that these protocol changes contribute to the development of treatments and the general health of patients. This work summarizes through bibliographic research some ideas of authors that approach on telemedicine. It is expected, due to the culture of convergence, the patient's autonomy, as for example, in choosing which form he wants to be consulted, which method offers him less risk and greater health benefit.
\end{abstract}

\section{INTRODUÇÃO}

As novas tecnologias de informação e comunicação (NTIC) ${ }^{1}$ convergiram para a oferta de novos produtos na medicina. Ultrapassando obstáculos em que a distância é o principal problema, a telemedicina surge como um aliado para a ampliação da atenção e da cobertura de cuidados com a saúde.

O uso da tecnologia de comunicação, como ligações telefônicas convencionais, mensagens ou videochamadas é muito utilizado por médicos em benefício de seus pacientes. Gradativamente são introduzidas novas técnicas que

1 Termo usado para as novas tecnologias e métodos para comunicar surgidos no contexto da Revolução Informacional, Revolução Telemática ou Terceira Revolução Industrial, desenvolvidas gradativamente desde a segunda metade da década de 1970 e, principalmente, nos anos 1990 (MORIGI; PAVAN, 2004). potencializam a informação e comunicação, também melhoram o entrosamento entre os profissionais médicos, e entre médicos e os pacientes. A telemedicina é o exercício da medicina à distância, cujas intervenções, diagnósticos, decisões de tratamentos e recomendações estão baseados em resultados de exames laboratoriais, anamnese ${ }^{2}$, prontuários e documentos transmitidos através de sistemas de telecomunicação (AMM, 1999).

A história do serviço de assistência médica à distância por meio da comunicação e da tecnologia remonta à época em que os aparelhos eletrônicos foram inicialmente introduzidos na sociedade (MATUSITZ; BREEN, 2007). O serviço de

2 Etimologicamente, a palavra anamnese vem do grego anamnesis, e significa recordar. A anamnese, na prática clínica, consiste na rememoração dos eventos pregressos relacionados à saúde, e na identificação dos sintomas e sinais atuais, no intuito principal de fazer entender, com a maior precisão possível, a história da doença que traz o paciente à consulta (SANTOS, 1999). 
telemedicina acontece há 60 anos, quando alguns psiquiatras, em 1959, usando rede por micro-ondas, consultaram pessoas há 112 milhas de distância do instituto médico que estavam alojados (PEREDNIA; ALLEN, 1995). No mesmo ano, exames telefluoroscópicos foram transmitidos via cabo coaxial. Em 1969, a Administração Nacional de Aeronáutica e Espaço (NASA) desempenhou um papel importante no desenvolvimento da telemedicina quando os astronautas começaram a voar no espaço. As condições fisiológicas da tripulação eram relatadas por meio de satélites de comunicação tanto da espaçonave quanto das roupas que os astronautas usavam durante as missões (AUSSERESSES, 1995).

No Brasil, a resolução CFM n. 1.643 de 26 de agosto de 2002 (BRASIL, 2002) autoriza a realização do uso da telemedicina entre médicos, conhecida como teleinterconsultas, quando um médico auxilia o outro com uma segunda opinião. Com o uso de tecnologia interativa, visando a assistência, educação e pesquisa.

Nesse sentido, a cibernização de consultas médicas e a convergência para serviços on-line apresentam-se positivamente em função de seu potencial inovador para os diversos atores envolvidos e mesmo para a saúde da população em geral (MALDONADO et al., 2016). Esses serviços aliaram a tecnologia de outras áreas com a medicina (MACHADO et al., 2010), resultando em um ambiente interdisciplinar e democrático para acessar os serviços de saúde, por integrarem regiões remotas à hospitais e centros de referência, objetivando a prevenção, diagnóstico e tratamento (MALDONADO et al., 2016).

Estudos enfatizam que a telemedicina é a junção de profissionais de saúde, tecnologia e comunicação (MIRANDA; ARAÚJO, 2012). Englobando ações de gestão e planejamento, pesquisa e desenvolvimento de conceitos e soluções em educação, assistência e pesquisa científica em saúde, aspectos éticos e legais (MALDONADO et al. 2016).

Além do mais, com o acesso rápido pela telemedicina pode-se economizar tempo e dinheiro, como a interação instantânea entre médico e paciente, melhor conforto, segurança e satisfação, custos mais baixos e alocação geográfica mais ampla de recursos (LOPES; HEIMANN, 2016). Por outro lado, há questões sérias que obstruem ou ameaçam seu crescimento e implementação de várias maneiras, desafios como a regulamentação ${ }^{3}$, privacidade do paciente, resistência de profissionais de saúde em adotar o serviço e investimento em operacional tecnológico para a efetiva prática.

É importante discorrer sobre a comunicação na

${ }^{3}$ Estabelecimento de um aparato que dê conta dos direitos dos pacientes, dos deveres do provedor de saúde (institucional ou telemedicina. No contexto, a mensagem torna-se a mudança do padrão de comunicação dos serviços de saúde. Com uma ligação telefônica, envio instantâneo de imagens, vídeos, áudios e mensagens de texto podem-se reduzir longas esperas por consultas médicas presenciais, diminuir a angústia e o medo do diagnóstico, acelerando o processo de cura da enfermidade, ou mesmo de atitudes favoráveis de mudanças de estilos de vida, que proporcionarão qualidade de vida à pessoa assistida.

Algumas pessoas que buscam serviços de saúde costumam previamente consultar o Google, webmd.com ou outras ferramentas de busca sobre qualquer sintoma que estejam apresentando (FUENTES, 2013). Muitas chegam até a acertar o diagnóstico. Utilizam da inteligência coletiva para ler, perguntar e compartilhar experiências sobre problemas de saúde em fóruns on line, aplicativos e outras mídias.

Seguindo o embasamento conceitual do autor Henry Jenkins (2009, p.41), quando define meios de comunicação "um meio é uma tecnologia que permite a comunicação e é um conjunto de protocolos que cresceram em torno de tecnologia, sendo mutáveis o conteúdo, o público e o status social". Observa-se que o uso da informação em saúde, passou por uma mudança de protocolos das pessoas que buscam esclarecimentos sobre saúde. Com a facilidade de acesso a informações, a clássica relação paternalista médicopaciente tomou outras proporções. O paciente e/ou seu acompanhante buscam compreender o quadro clínico, eles leem sobre o assunto, querem receber informações claras e imparciais para tomar as decisões de saúde juntamente com o médico (SAY; THOMSON, 2003). Isso se torna importante para o consentimento informado e para a tomada de decisão compartilhada. Assim, podem se submeter a exames e tratamentos sabendo dos riscos, das incertezas, do tempo que perdurará determinado problema de saúde, de diagnósticos falso-positivos, dos benefícios. E o modo como essas mudanças de protocolo evoluem poderá determinar desdobramentos de tratamentos e da saúde geral dos pacientes.

Nesse sentido, questiona-se como a comunicação pode contribuir no serviço de telemedicina? Como a inteligência coletiva por meio de experiências reais de vida pode contribuir na convergência da medicina tradicional para a telemedicina? Propõe-se com este objeto de estudo discorrer sobre o papel do profissional jornalista em promover a informação em saúde, principalmente em serviço de telemedicina, contribuindo para a autonomia do paciente, como por exemplo, em escolher qual forma deseja ser consultado, qual método que lhe oferece menor risco e maior

profissional) e dos sistemas de informação utilizados (MALDONADO et al., 2016). 
benefício de saúde.

\section{METODOLOGIA}

Esta discussão baseia-se em conceitos de comunicação apontados na obra de Henry Jenkins (2009), tendo em vista discutir a telemedicina sob o prisma da mudança de protocolos das pessoas que buscam esclarecimentos sobre saúde.

Portanto, este artigo é construído através da análise observacional do serviço (telemedicina) em foco. Este debate propulsiona um grande desafio na comunicação em saúde, que pode influenciar na percepção pública e em cuidados de saúde.

Nesta discussão, supõe-se que essas mudanças de protocolo contribuem para desdobramentos de tratamentos e da saúde geral dos pacientes. Para tal, discorrer-se-á sobre o papel do profissional jornalista em promover a informação em saúde, principalmente em serviço de telemedicina.

\section{COMUNICAÇÃO}

A comunicação entre profissionais de saúde e pessoas assistidas em serviços de saúde, costuma definir a preferência do público atendido por aquele profissional de saúde que enxergue o usuário como sujeito, não somente como um corpo, um prontuário ou um número de atendimento. $\mathrm{O}$ vínculo e a tomada de decisões compartilhadas, via de regra, são fundamentais para a adesão e a continuidade do tratamento (SCHIMITH et al., 2011).

Assim, a Política Nacional de Atenção Básica (BRASIL, 2012, p. 21) define vínculo como a construção de relações de afetividade e confiança entre o usuário e o trabalhador da saúde, o que permite o aprofundamento do processo de corresponsabilização pela saúde, construído ao longo do tempo, além de carregar, em si, um potencial terapêutico.

\section{Relação médico-paciente}

A relação médico-paciente é defendida por Gadamer (1994, apud CAPRARA et al., 1999) como a humanização do atendimento ao paciente, desde o diagnóstico até a terapia, compreendendo uma relação de confiança e colaboração médico-paciente. Para que haja a efetiva promoção da saúde, garantindo a integridade física, psíquica e social do paciente (CAPRARA et al., 1999).

Nesse contexto, vale lembrar, o novo perfil de pacientes. São pessoas com acesso às informações, via internet, redes sociais, canais de notícias, tornando-se potencialmente poderosos (GARBIN et al., 2008). O que pode influenciar ou ainda, modificar o relacionamento de médicos com seus pacientes. Em geral, nessa nova relação médico-paciente:

\begin{abstract}
Os profissionais devem trabalhar com o paciente, ao invés de "para ele", usando mais tempo para escutar, absorver e valorizar as necessidades cognitivas, sociais e emocionais de seus pacientes. 0 médico deve valorizar a vida do paciente, e não somente seu corpo. Assim, deve fornecer informações de boa qualidade, discutir questões referentes ao diagnóstico, tratamento e resultados, respeitando os desejos do paciente em relação à tomada de decisões. Para tal, é fundamental que os próprios médicos se mantenham informados e atualizados (GARBIN et al., 2008).
\end{abstract}

O ideal é que médicos e pacientes interajam de maneira que exista o livre consentimento informado. Os pacientes devem ser informados sobre os prós e contras de um tratamento e suas alternativas, e devem decidir, com base nisso, se desejam se submeter ao tratamento (GIGERENZER et al., 2007). O consentimento informado não é simplesmente obter o consentimento dos pacientes (MOUMJID, et al., 2007). Dado que, estudos indicam que os médicos raramente comunicam as incertezas sobre os riscos e benefícios dos tratamentos para os pacientes (BRADDOCK III et al., 1999).

\section{CULTURA DA CONVERGÊNCIA}

\section{Inteligência coletiva}

Um grande número de consumidores de comunicação online está surgindo substancialmente e está se formando um canal comunitário virtual através do qual pessoas têm buscado, e ao mesmo tempo, fornecido informações relacionadas à saúde (MATUSITZ; BREEN, 2007).

As fontes são numerosos fóruns, aplicativos, buscadores, comunidades e grupos on-line onde os participantes relatam experiências, positivas ou negativas, sobre clínicas, hospitais, institutos, profissionais de saúde, terapias, medicamentos, doenças, entre outros assuntos (FUENTES, 2013).

Segundo um levantamento intitulado Jornada Digital do Paciente (2016) foram entrevistados 3.860 usuários, com perguntas sobre saúde e bem-estar. $90 \%$ dos participantes disseram que buscam informações antes e/ou depois de uma consulta médica. $94 \%$ deles se informam na internet. Esses dados foram mais comuns entre os jovens, que talvez não estavam seguros com as informações passadas pelos 
profissionais.

Tal como Jenkins (2009, p.88), quando descreveu a anatomia de uma comunidade de conhecimento, essas pessoas que participam desses ambientes de inteligência coletiva frequentemente sentem a necessidade de demonstrar ou documentar como sabem, o que sabem e o conhecimento proveniente da experiência real de vida.

A inteligência coletiva em assuntos de saúde pode ser representada por consumidores informados que usam a internet para coletar informações sobre uma condição médica de especial interesse para eles, Ferguson (2007) denominou essas pessoas de e-pacientes, ou seja, pessoas que procuram informações na web sobre saúde, seja para si próprios ou para amigos e familiares.

É bem verdade que as pessoas estão mais esclarecidas sobre assuntos de saúde, não aceitam somente a opinião médica, buscam artigos, leem a bula do medicamento, consultam outros especialistas (GARBIN et al., 2008), acessam extensões tecnológicas que Ihes permitam de fato, decidir sobre sua condição clínica.

Quando Mcluhan (1969, p. 19) observou as tecnologias como uma extensão do homem, ele mencionou a ação de "falar ao telefone", dando a dimensão de um grande ambiente, o meio. Comparando à inteligência coletiva em saúde, observa-se que o real impacto da interação dessas pessoas, está na cultura que se desenvolve nesse meio para se chegar até a mensagem.

O monopólio do conhecimento na área médica representa um desafio ao livre acesso à informação (FREIDSON, 1989 apud GARBIN, 2008). Uma vez observado o processo de como esse meio é cultivado, alguns profissionais médicos receiam uma redução de sua posição social e da confiança dos seus pacientes, resultando na busca por terapias alternativas (HARDEY, 1999). Em par e par à visão de Mcluhan (1969, p. 19), ele aponta:

\footnotetext{
As sociedades sempre foram moldadas pelos meios que os homens usam para comunicar-se, do que pelo conteúdo da comunicação. O alfabeto por exemplo é uma tecnologia absorvida pelas crianças de tenra idade de maneira inteiramente inconsciente quase se poderia dizer por osmose. [...] A tecnologia elétrica fomenta e encoraja o envolvimento e integração (MCLUHAN, 1969, p. 19).
}

Por outro lado, ressalta-se as dinâmicas não lineares de busca de informações na internet sujeitas aos efeitos da hiperinformação, como o excesso de opções disponíveis. Para o autor Carlos Ricardo (2005), "o meio" - a internet, passa por reformas organizacionais, as quais serão capazes de reorganizar a lógica de trabalho e descentralizá-la:

\begin{abstract}
A internet poderá estar na vanguarda duma visão muito diferente do trabalho e da sociedade. Esta visão assenta numa forma de organização, naturalmente concebida para se difundir, que passa por uma estrutura de responsabilidade e de autoridade mais descentralizada (RICARDO, 2005)
\end{abstract}

Nessa perspectiva, as consequências da tecnologia no meio são inevitáveis. O que realmente perpassa ao controle é o planejamento do caminho dessa informação, mesmo que não seja absorvida da forma como foi planejada, essa informação pode ser estruturada pelo comunicólogo para traçar seus objetivos e evitar possíveis erros em seu trajeto. Dado ao fato de que profissionais jornalistas exercem influência poderosa na percepção pública da saúde e dos cuidados de saúde, quando propagam informações importantes sobre doenças, prevenção, controle etc.

É sabido que muitos sites também representam um desserviço à saúde pública. A exemplo disso são os falsos dados e notícias, muitas vezes sob a forma de pacientes que descrevem respostas milagrosas a um novo medicamento ou relatos de danos de medicamentos (GIGERENZER et al., 2007).

\section{CONVERGÊNCIA DA MEDICINA}

A tecnologia é cotidianamente observável, ela está inserida em roupas, consultas médicas, serviços bancários, e daqui a um futuro próximo, estará embutida nos corpos humanos. As telecomunicações e a medicina (de fato, já se pratica a telemedicina) são hoje exemplos reais da magnanimidade tecnológica (SQUIRRA, 2012).

De acordo com Silva et al. (2010), métodos e intervenções com tecnologia contribuem para a promoção da saúde, prevenção, diagnóstico e tratamento de doenças, além de cuidados a longo prazo. Razão pela qual o serviço de telemedicina surge, com profissionais que atendem pacientes em locais remotos usando apenas um smartphone ou computador (PEREDNIA; ALLEN, 1995). Machado et al. (2010) complementa:

A telemedicina contribui na prestação da assistência médica a pacientes que estão geograficamente distantes, além de difundir cuidados na área da saúde para localidades desprovidas desses serviços. 
Trata-se de serviço emergente que apresentou contribuições facilitando o processo assistencial de melhor qualidade, obtenção de uma segunda opinião médica a custos acessíveis. Um dos resultados desse tipo de proposta é a diminuição do deslocamento dos pacientes para serem atendidos, contribuindo para a descentralização e diminuição da demanda de atendimento nos hospitais públicos da capital (MACHADO et al., 2010).

Todavia, a telemedicina requer normatização principalmente por questões éticas e legais. Tornando-se a posteriori em mais uma alternativa de saúde para transpor barreiras geográficas e estabelecer propostas de procedimentos de alta complexidade em favor de comunidades hoje ainda tão desassistidas (FRANÇA, 2009).

Para isso, é preciso capacitar os recursos humanos nas múltiplas etapas da telemedicina (LOPES; HEIMANN, 2016). Nesse ínterim, destaca-se o papel do profissional da comunicação social. Ele poderá atuar na disponibilização de informações fidedignas de saúde na internet. Possibilitando o acesso a dados, produzindo conteúdos para blogs, fóruns, comunidades on-line que relatem as queixas fornecidas por pacientes e profissionais da saúde.

Dessa forma, Miranda e Araújo (2012) preocupam-se com a qualidade dos conteúdos relativos à saúde divulgados na internet. Para os autores, tais conteúdos são oriundos de instâncias não comprometidas em avaliar a informação antes de torná-la pública. Eles ponderam a necessidade de se estabelecer sanções penais em casos de publicações danosas ou fraudulenta de informação.

Por este motivo, é importante que um profissional habilitado na produção de conteúdo faça uma apuração deontológica das informações e respectivos riscos quanto à publicação inadequada de produtos midiáticos relativos à saúde.

\section{CONSIDERAÇÕES FINAIS}

A cibernização e a convergência para serviços on-line representa um verdadeiro desafio para os profissionais envolvidos e para o sistema de saúde. A telemedicina agrupa recursos tecnológicos originários de outras áreas.

Nesse sentido, profissionais de comunicação social são imprescindíveis na produção de conteúdo para a telemedicina. Em razão de exercerem influência poderosa na percepção pública e nos cuidados de saúde, quando propagam informações importantes sobre doenças, prevenção, controle etc.

O jornalista poderá atuar com telemedicina disponibilizando informações fidedignas, colaborando para a troca de informações entre médicos-pacientes e profissionais de saúde, independentemente da distância. Por meio da apuração deontológica das informações e respectivos riscos quanto à publicação inadequada de produtos midiáticos relativos à saúde.

\section{REFERÊNCIAS}

Associação Médica Mundial-AMM. Declaração de Tel Aviv sobre Responsabilidades e Normas Éticas na Utilização de Telemedicina. 51 a Assembléia Geral da Associação Médica Mundial. Disponível em http://www. unifesp. br/dis/set/law/declaracaotelaviv. html, 1999.

AUSSERESSES, A. D. Telecommunications requirements for telemedicine. Journal of medical systems, v. 19, n. 2, p. 143-151, 1995.

BRASIL. Conselho Federal de Medicina n. 1.643 de 26 de agosto de 2002.

BRASIL. Ministério da Saúde. Secretaria de Atenção à Saúde. Departamento de Atenção Básica. Política Nacional de Atenção Básica. Brasília: Ministério da Saúde, 2012. (Série E. Legislação em Saúde).

BRADDOCK III, C. H., EDWARDS, K. A., HASENBERG, N. M., LAIDLEY, T. L., \& LEVINSON, W. (1999). Informed decision making in outpatient practice: time to get back to basics. Jama, v. 282, n. 24, p. 2313-2320, 1999.

FERGUSON, T. e-Patients: How they can help us h/eal health care, 2007. Disponível em: <http://e-patients.net/e/Patients_White_Paper.pdf>. Acesso em: 03 outubro de 2019.

FRANÇA, G. V. Telemedicina: breves considerações éticolegais. Revista Bioética, v. 8, n. 1, 2009.

FREIDSON, E.A. Medical work in America: essays on health care. New Haven: Yale University Press, p.178-205, 1989 apud GARBIN, Helena Beatriz da Rocha et al. A internet, o paciente expert e a prática médica: uma análise bibliográfica. Interface-Comunicação, Saúde, Educação, v. 12, p. 579-588, 2008.

FUENTES, S. El lugar de Internet en las búsquedas de salud. Humanidades y Ciencias de la Educación, Almanaque 03, julho de 2013.

GADAMER, H. G. Dove si Nasconde la Salute. Milano: Raffaelo Cortina Editore, 1994 apud CAPRARA, Andrea et al. A relação paciente-médico: para uma humanização da prática médica, 1999.

GARBIN, H. B. D. R., PEREIRA NETO, A. D. F., \& GUILAM, M. C. R. A internet, o paciente expert e a prática médica: uma 
análise bibliográfica. Interface-Comunicação, Saúde, Educação, v. 12, p. 579-588, 2008.

GIGERENZER, G., GAISSMAIER, W., KURZ-MILCKE, E., SCHWARTZ, L. M., \& WOLOSHIN, S. Helping doctors and patients make sense of health statistics. Psychological science in the public interest, v. 8, n. 2, p. 53-96, 2007.

HARDEY, M. Doctor in the house: the Internet as a source of lay health knowledge and the challenge to expertise. Sociology of Health \& Illness, v. 21, n. 6, p. 820-835, 1999. JENKINS, H. Cultura da Convergência. 2 a ed. São Paulo: Aleph, 2009.

JORNADA DIGITAL DO PACIENTE, 2016. Disponível em: https://saudebusiness.com/. Acesso em 08/06/2019, às $16: 36$.

LOPES, J. E.; HEIMANN, C. Uso das tecnologias da informação e comunicação nas ações médicas a distância: um caminho promissor a ser investido na saúde pública. Journal of Health Informatics, v. 8, n. 1, 2016.

MACHADO, F. S. N., CARVALHO, M. A. P. D., MATARESI, A., MENDONÇA, E. T., CARDOSO, L. M., YOGI, M. S., ... \& SALAZAR, M. Utilização da telemedicina como estratégia de promoção de saúde em comunidades ribeirinhas da Amazônia: experiência de trabalho interdisciplinar, integrando as diretrizes do SUS Ciência \& Saúde Coletiva, v. 15, p. 247-254, 2010.

MALDONADO, J. M. S. D. V., MARQUES, A. B., \& CRUZ, A. Telemedicina: desafios à sua difusão no Brasil. Cadernos de Saúde Pública, v. 32, e00155615, 2016.

MATUSITZ, J.; BREEN, G.M. Telemedicine: Its effects on health communication. Health communication, v. 21, n. 1, p. 7383, 2007.

MCLUHAN, M. O meio é a mensagem. MORTENSEN, C. David. Teoria da, 1969.

MIRANDA, R. C.; ARAUJO, T. C. C. F. Alcances e limites das tecnologias de informação e comunicação em saúde: um estudo com profissionais da área. Revista da SBPH, v. 15, n. 2, p. 33-45, 2012.

MORIGI, V. J.; PAVAN, C. Tecnologias de informação e comunicação: novas sociabilidades nas bibliotecas universitárias. Ciência da informação. Brasília. Vol. 33, n. 1, p. 117-125, 2004.

MOUMJID, N., GAFNI, A., BREMOND, A., \& CARRERE, M. O. Shared decision making in the medical encounter: are we all talking about the same thing? Medical Decision Making, v. 27, n. 5, p. 539-546, 2007.

PEREDNIA, D. A.; ALLEN, A. Telemedicine technology and clinical applications. Jama, v. 273, n. 6, p. 483-488, 1995.

RICARDO, C. A evolução tecnológica e a mudança organizacional. VISÕES DISCIPLINARES, p. 49, 2005.

SANTOS, J. B. Ouvir o paciente: a anamnese no diagnóstico clínico. Brasília méd, v. 36, n. 3/4, p. 90-95, 1999.
SAY, R. E.; THOMSON, R. The importance of patient preferences in treatment decisions-challenges for doctors. Bmj, v. 327, n. 7414, p. 542-545, 2003.

SCHIMITH, M. D., SIMON, B. S., BRÊTAS, A. C. P., \& BUDÓ, M. D. L. D. Relações entre profissionais de saúde e usuários durante as práticas em saúde. Trabalho, Educação e Saúde, v. 9, n. 3, p. 479-503, 2011.

VIANA, A. L., \& Silva, H. P. D. Avaliando a difusão de tecnologias médicas no sistema de saúde privado no Brasil: o caso da tomografia por emissão de pósitrons (PET). Rev. bras. saúde matern. infant, p. s187-s200, 2010.

SQUIRRA, S. A cibercomunicação em um wikipaper colaborativo. Ciber mídias: extensões comunicativas, expansões humanas. Porto Alegre: Buqui, 2012.

Submissão: 13/03/2020

Aprovado para publicação: 25/03/2020 